\title{
Formación de profesores en Educación Física en Brasil: comprensión de estudiantes noveles de la Universidad Federal de San Carlos
}

\author{
Teachers' formation of physical education in brazil: undestandig of new students of san \\ carlos federal university
}

\section{A formação de professores de Educação Física no Brasil: compreensões de estudantes recém-ingressados na Universidade Federal de São Carlos}

\author{
Luiz Gonçalves Junior, ${ }^{a}$ Fábio Lemos, ${ }^{b}$ Denise Corrêa, ${ }^{c}$ Sergio Toro ${ }^{d}$
}

\author{
${ }^{a}$ Departamento de Educacon Física y Motricidad Humana, Universidad Federal de San Carlos. Sociedad de \\ Pesquisa Cualitativa en Motricidad Humana. Correo electrónico: luiz@ufscar.br. \\ ${ }^{b}$ Centro de Educación Universitaria Claretiano. Sociedad de Pesquisa Cualitativa en Motricidad Humana. \\ Correo electrónico: fabiomizuno@claretiano.edu.br. \\ ${ }^{c}$ Univesidad Estadual Paulista-Bauru. Sociedad de Pesquisa Cualitativa en Motricidad Humana. \\ Correo electrónico: decorrea@fc.unesp.br \\ ${ }^{d}$ Universidad Austral de Chile. Sociedad de Pesquisa Cualitativa en Motricidad Humana. \\ Correo electrónico: seatoro@gmail.com
}

\begin{abstract}
RESUMEN
La frecuente caracterización de la Licenciatura en Educación Física, asociada a aspectos técnicos deportivos y de entrenamiento, es el motivo este estudio. El objetivo es comprender las razones a la base de esta situación, en tal sentido se realizó paralelamente un estudio teórico sobre la formación de profesores en Educación Física en Brasil y una investigación empírica en estudiantes de primer año de esta licenciatura. Como instrumento de registro de las comprensiones de los estudiantes fue utilizada la pregunta abierta: “¿por qué usted escogió el curso de Licenciatura en Educación Física? Para comprender los datos se organizó la información en dos categorías temáticas: Categoría A No identificación con la enseñanza escolar; Categoría B Motivos en la (falta) elección profesional. Las respuestas permitieron comprender que la elección de la licenciatura se configura mayoritariamene por falta de opción, que por una real identificación profesional y de procesos reflexivos para la transformación social.
\end{abstract}

Palabras claves: formación de profesores, educación física, elección profesional.

\begin{abstract}
The frequent characterization about the Physical Education Degree is associated to technical-sport and training aspects and this is the reason of this study. The objective is to understand the motives to the basis of this situation. In this sense, a theoretical study was developed regarding the Physical Education Teaching Training in Brazil; at the same time, an empirical investigation was carried out with first-year students of the cited degree. The register tool to collect the students' understandings was an open question expressed as: "Why did you choose the course of Physical Education Degree?" which was answered in written form by the students. So as to make up an understanding of the data collected, the information was organized in two thematic categories; Category A: no identification with schooling; Category B: reasons in relation to the (lack) of professional vocation. Based on the students' answers, it was possible to comprehend that choosing the mentioned degree it is mostly set up by a lack of option, instead of a real professional identity and thoughtful processes of transformation in society.
\end{abstract}

Key words: teaching training, physical education, professional choice. 


\section{RESUMO}

A frequente descaracterização da Licenciatura em Educação Física, associada a aspectos técnico-esportivos e de treinamento, motivou o estudo que objetivou compreender os motivos que baseiam tal situação. Para tanto, realizou-se um estudo teórico sobre a formação de professores de Educação Física no Brasil, juntamente com uma pesquisa empírica realizada com estudantes do primeiro ano da citada Licenciatura. Como instrumento de registro das compreensões dos discentes, foi utilizado o questionário contendo uma questão aberta: "Por que você escolheu o Curso de Licenciatura em Educação Física?”, o qual foi respondido pelos estudantes por escrito. Para estabelecer uma compreensão dos dados obtidos, as informações foram organizadas em categorias temáticas: Categoria A: Não identificação com o ensino escolar; Categoria B: Motivos para a (falta de) escolha profissional. A partir das respostas dos estudantes pode-se compreender que a escolha pela mencionda Licenciatura configurase mais como falta de escolha profissional do que como uma real identificação profissional e de processos reflexivos para a transformação social.

Palavras-chave: formação de professores, educação física, escolha profissional.

\section{INTRODUCCIÓN}

En el derrotero de iniciar transformaciones sutantivas dentro de la formación de profesionales de la educación, se hace imprescindible conocer las auténticas intencionalidades o motivaciones que llevan a un joven a iniciar un proceso de construcción profesional dentro de la pedagogía. Específicamente, en nuestro cotidiano pedagógico, orientado hacia la docencia en la enseñanza superior de la Licenciatura en Educación Física, hemos presenciado, a lo largo de más de una década, la confusión de los estudiantes de primer año cuando justifican los motivos de la elección de esta carrera. Entre las respuestas comúnmente verbalizadas al inicio del curso están: el gusto por la práctica de actividades deportivas y la afinidad con el ambiente de las academias (gimnasios o centros de fitnes en Brasil) y de los clubes pensando en sus futuras actuaciones profesionales, las que, en el contexto brasilero, están relacionadas al campo de atuacion del Bachiller'.

Tal duda en la exposición de las intencionalidades nos han causado extrañeza, pues la información sobre la caracteristicas de la Licenciatura en Educación Física, para cada proceso selectivo de ingreso de los/as estudiantes, son divulgadas en los Manuales del Candidato y en los prospectos de los cursos. Sin embargo, en los discursos de los estudiantes ingresantes poco destacan el aprecio por la Educación Física que tuvieron en la escuela y/o el interés de desempeñarse profesionalmente en tal contexto. Esto nos indica que al optar por la carrera los estudiantes de cada nueva cohorte de ingreso no tienen una clara comprensión sobre la Licenciatura, y que apesar del buen nivel de formación escolar, dada la aprobación de ingreso en procesos selectivos tipicamente rigurosos para el logro de cupos en universidades públicas, están asistiendo a un curso que, en última instancia, no converge con sus intereses momentáneos, sobre todo en la formación deliberada para el desempeño profesional.

Es importante enfatizar que estamos considerando que los saberes relacionados a lo que es ser docente también serán movilizados con la propia formación académica y, siendo así, no

En Brasil, los cursos de Licenciatura preparan exclusivamente profesores para desempeñarse en el sistema escolar y los cursos de Bachiller preparan exclusivamente profesionales para el desempeño en ambientes no escolares. En el caso específico del Bachiller en Educación Física, la formación profesional se centra para la posterior actuacion en clubes, academias (gimnasios de fitnes), campamentos, centros de salud y sitemas afines. Observamos también que hay legislación específica y fiscalización del desempeño por parte de los Consejos Regionales de Educación Física (CREF). 
esperamos que los estudiantes novatos de los cursos de Licenciatura Educación Física hayan adquirido tales saberes luego de los primeros días de estudio. Al contrario, comprendemos que las bases de los saberes profesionales son construídas al inicio de la carrera como desempeño, más que en los primeros semestres de estudio (Tardif y Raymond, 2000).

Para Tardif y Raymond (2000), el inicio de la carrera constituye también una fase crítica en relación a las experiencias anteriores y a los reajustes, epistémicos y metodológicos, que se realizarán en función de las realidades del desempeño laboral.

(...) ese processo está ligado tambien a la socialización profesional del profesor y al que numerosos autores llamarán de "choque con la realidad", "choque de transición" o aún "choque cultural", nociones que remiten a la confrontación inicial con la dura y compleja realidad del ejercicio de la profesión, a la desilusión y al desencanto de los primeros tiempos de profesión y, de manera general, a la transición de la vida de estudiante hacia la vida más exigente de trabajo (Tardif y Raymond, 2000: 226).

Hay, entonces, dos fases durante los primeros años de carrera:

1) Una fase de exploración (del primero al tercer año) en la cual el profesor hace una elección provisoria de su profesión.

(...) se inicia a través de tentativas y errores, siente la necesidad de ser aceptado por su círculo profesional (alumnos, colegas, directores de escuela, padres y apoderados etc.) y experimenta diferentes roles. (...) Esa fase es tan crucial que lleva un porcentaje importante (...) de novatos a abandonar la profesión o simplemente a cuestionarse sobre la elección de la profesión y sobre la continuidad de la carrera, conforme la magnitud del "choque con la realidad" (Tardif y Raymond, 2000: 227-228).

2) La fase de estabilización y de consolidación (del tercer al séptimo año), en que el profesor invierte, en un largo plazo, en su profesión y los otros miembros de la organización reconocen que es capaz de lograrlo.

Esa fase se caracteriza también por una confianza mayor del profesor en sí mismo (y también de los otros agentes en el profesor), por el dominio de los diversos aspectos del trabajo, principalmente los aspectos pedagógicos (gestión de la clase, planificación de la enseñanza, asimilación personal de los programas etc.), lo que se manifiesta en un mejor equilibrio profesional (...) el profesor está menos centrado en sí mismo y en la materia y más en los alumnos (Tardif y Raymond, 2000: 228).

Todavía el parametro calcado en el tiempo cronológico no significa que los eventos vayan ocurriendo naturalmente, se desencadenan no sólo en función de los meses recorridos desde el inicio de la carrera, sino en función de los acontecimientos sustantivos que marcan la trayectoria profesional, incluyendo las condiciones de ejercicio de la profesión (Tardif y Raymond, 2000).

Se entiende la nebulosa comprensión que los aspirantes a profesores pueden presentar durante la formación inicial e, inclusive, cargan al principio de la carrera, pero lo que estamos resaltando es que en el caso de la formación profesional en Educación Física en Brasil, esa comprensión remite hacia el área de actuación del Bachiller. Frente a tal problemática se tornó relevante para la investigación los posibles motivos a la base de tal comprensión de los estudiantes recien ingresados al curso de Licenciatura en Educación Física. De esta forma, asociamos la pesquisa teórica que permite contextualizar la formación profesional en Educación Física en Brasil, con el trabajo de campo junto a estudiantes del citado curso en la Universidad Federal de São Carlos (UFSCar). 


\section{FORMACIÓN PROFESIONAL EN EDUCACIÓN FÍSICA EN BRASIL}

De acuerdo con Ramos (1995: 12), las instituciones para formación de recursos humanos en Educación Física obedecen a la siguiente cronología:

(...) en 1909, es creada la Escuela de Educación Física de la Fuerza Policial de São Paulo; en 1914, se funda la Liga de Deportes de Marinha; el 10 de enero de 1922, por ordenanza del Ministerio de Guerra, se crea el Centro Militar de Educación Física, ligado a la Escuela de Sargentos de Infantería; el 19 de octubre de 1933, el Centro Militar de Educación Física, por medio del Decreto 23.252, se transforma en la Escuela de Educación Física del Ejército; en 1934, la primera escuela civil de Educación Física es creada por el Gobierno del Estado de São Paulo (Decreto 4.855, del 27 de enero de 1931); en 1939, es fundada la Escuela Nacional de Educación Física y Deportes de la Universidad del Brasil (Decreto-Lei 1.212).

Con la creación de la Escuela Nacional de Educación Física y Deportes de la Universidad del Brasil, en 1939, surge el primer curso de Educación Física vinculado a una universidad. Segun Oliveira, Betti y Oliveira (1988: 47), "La legislación que fundó la Escuela Nacional exigió, a partir de entonces, la posesión del diploma de curso superior para el ejercicio del magisterio en Educación Física en los establecimentos de enseñanza superior, secundario, profesional y normal".

La regulación de la formación de profesores ocurrió, apesar de que las primeras escuelas superiores en Educación Física en Brasil datan desde el inicio del Siglo XX, solamente en el año de 1969, a través del "Parecer 894/69 y Resolução 69/69 (del Consejo Federal de Educación), que fijó el currículo mínimo en los cursos superiores” (Ramos, 1995: 15).

Observamos que antes de la citada resolución:

(...) bastaba el antiguo primer grado, actual enseñanza fundamental (básica), para tener acceso al curso de Educación Física. Pero incluso tal resolución se presentó como una disminución de la formación universitaria, pues determinaba la graduación en Educación Física solo en nivel de licenciatura y en rápidos tres años, con un mínimo de 1880 horas/aula. Concomitantemente, aún era posíble para el graduando titularse como "Técnico Deportivo", bastando para eso cursar dos disciplinas práctico-deportivas adicionales, ofrecidas por la facultad (Gonçalves Junior, Ramos y Machado, 2001: 1).

Esta estrutura curricular se mantuvo inalterada hasta que el Parecer CFE 215/87 y la Resolución CFE 3/87, del 16 de junho de 1987, permitieron proponer la estruturación curricular de los cursos superiores de Educación Física, alterando/modificando la Licenciatura y abriendo la posibilidad de creación del Bachillerato en el área (Ramos, 1995). Tales mudanzas en la estruturación curricular de los cursos superiores de Educación Física no ocurrieron por casualidad. Andrade Filho (2001: 27) recuerda que el advenimiento del fenómeno de deportivizacion ${ }^{2}$ en la sociedad,

(...) alteró substancialmente los hábitos de vida de la población mundial, en particular de la población brasileña, después de los años 70, diversificando y expandiendo la demanda y la oferta

"Proyecto que se revitalizó a partir del discurso de los medios de comunicación sobre el desempeño de Brasil en los Jeuegos Olímpicos de Sidney, 2000, considerado insatisfactorio; [...] la Educación Física continúa siendo considerada como base para la formación de atletas, colocada en posición subalterna al deporte espectáculo" (Betti, 2009: 218). 
de actividades físicas no escolares, fundamentalmente en el sentido del ocio y de la salud. (...) como consecuencia de esto, los cursos de Educación Física fueron incorporando disciplinas científicas, pedagógicas y técnicas, acarreando la caracterización erronea de la especificidad de los currículos de licenciatura plena.

De esta manera, fue provocada una verdadera "inflación” en los currículos de los cursos de Licenciatura en Educación Física, a fin de atender a las nuevas áreas de actuación como musculación, gimnasia aeróbica y Educación Física adaptada (Betti y Betti, 1996).

Frente de esa nueva realidad, ampliación y diversificación del mercado de trabajo/ actuación profesional, las Instituciones de Enseñanza Superior (IES) en Educación Física comenzaron a manifestar interes por currículos más precisos, más estruturados, más volcados a la investigación y a delimitación del campo profesional específico de la Educación Física, apuntando al movimiento en defensa/valorización del Bachillerato:

Basado en el deseo manifestado por las Escuelas de Educación Física [...] quedó clara la necesidad de introducir el bachiller en el parecer y resolución que trata de la licenciatura, lo que se dió a través del parecer del Consejo Federal de Educación número 215/87, aprobado el 11 de março de 1987 (Tojal, 1995: 79).

Barros (1995: 71) considera la Resolución CFE 03/87, surguida del Parecer CFE 215/87, uno de los marcos de la transformación de la Educación Física, que representa una verdadera “(...) emancipación de la Educación Física en Brasil” y plantea:

Hasta 1987 todos los cursos de Graduación en Educación Física, diríamos, vestían una camisa de fuerza impuesta por CFE, que restringía sus posibilidades al ofrecimento del curso de licenciatura, y complementariamente, el de Técnico Deportivo. De este modo, limitaba la motivación de los estudiosos del área y la responsabilidad de las Instituciones de Enseñanza Superior involucradas en la preparación de esos profesionales. Esta situación no posibilitó a la Educación Física descubrirse a sí misma.

Destacamos que el área de Educación Física, con esa Resolución, según Oliveira y Costa (1999: 87) se tornó “(...) la primera del país en tener autonomía curricular en el nivel de graduación", fue la pionera en la eliminación del llamado currículo mínimo, lo que colaboró para el salto cualitativo en el área por la libertad de construcción curricular. Aun, de acuerdo con Oliveira y Costa (1999: 88), “(...) las Directrices organizadas para la Educación Física (Resolución 3/87) sirvieron de base para el Ministerio de Educación para recomendar a las demás áreas de conocimento".

Apesar de alguna mejora en la estruturación del currículo, originada con la Resolución 03/87, percibimos que apesar de la proposición representa un avance y contiene "idealmente" una formación más específica para la actuación de los profesionales egresados de los cursos de Educación Física, a saber: licenciados para el ambiente escolar (prioritariamente) y bachilleres para el ambiente no-escolar (prioritariamente), en la realidad ocurrirán algunas distorciones en la formulación de los currículos de los cursos de Educación Física que, principalmente en función de atender a "principios legales" y de "mercado de trabajo incierto", optaron por lo que se llamó "licenciatura ampliada", buscando agregar conocimientos de los ámbitos escolar y no-escolar (Gonçalves Junior, Ramos y Machado, 2001). 
Posterior a tal Resolución, el área contó con una Propuesta de Directrices Curriculares para los Cursos de Graduación en Educación Física, elaborada por la entonces Comisión de Especialistas de Enseñanza en Educación Física, que establecía dos años de formación básica y dos años de formación específica para Licenciatura o Bachillerato (Comissão de Especialistas de Ensino em Educação Física, 1999). Aunque tal propuesta no prosperó, tuvo como consecuencia la publicación de la legislación específica para todos los cursos de formación de profesores para la educación básica (Conselho Nacional de Educação. Brasil, 2002a, 2002b), la cual orienta el fortalecimiento de las Licenciaturas. Así, los cursos de Licenciatura, incluyendo el de Educación Física, pasaron a tener la denominación e integralidad própia, constituyéndose en currículos própios de Licenciatura que no se confundieran con el Bachillerato.

En el contexto de formación en Educación Física, se puede afirmar que se fue constituyendo una amplia oposición entre una aproximación científico-médica y deportiva, fundamentada principalmente en las ciencias "duras" y aplicadas, y una aproximación más ecuménica (integrando la contribuición diversificada de las ciencias sociales y humanas y de las ciencias de la educación), educativa y pedagógica (Borges, 2005). Esa oposición no está limitada a un debate de ideas, sino que se inserta en la própia estruturación del campo de la Educación Física, marcado por diferentes ideologías sobre su naturaleza y finalidad, sobre sus contenidos, sus bases de conocimientos y sobre los roles y las misiones que los docentes deben asumir y buscar. También se expresa en la división entre Bachillerato y Licenciatura (Borges, 2005).

En el papel, la Educación Física sería caracterizada por dos cursos distintos: Licenciatura, responsable por la formación de profesionales calificados para actuar en el ámbito de la Educación Física escolar, de la Educación Infantil a la Enseñanza Média; y Bachillerato, responsable por la formación de profesionales calificados para actuar fuera del ambiente escolar, o sea, en clubes, academias, campamentos, unidades de salud de la família etc.

En tanto, apesar de la visión que consideraba la creación del Bachillerato como una conquista, pues permitía consolidar un cuerpo de conocimiento que diese mayor legitimidad a propia profesión. Este cambio provocó una “división de águas” en la profesionalización y en la consolidación del área de conocimiento de la Educación Física (Benites, Souza Neto y Hunger, 2008).

Los autores que defendían la creación de cursos de Bachilerato sostenían que ese emprendimiento no sólo traería contribuciones en la calificación de los profesionales del área, sino que también ayudaría en la construcción de una producción científica relevante, elevando el nivel de los cursos y, consecuentemente, proporcionaría su reconocimiento en las comunidades académicas y sociales. Por otro lado, y reconociendo la presión producida de la ampliación del mercado de trabajo y la propia fragilidade de los cursos que formaban profesores de Educación Física, los autores contrários a la creación del Bachillerato sostenían que esto causaría una fragmentación aún mayor en la formación de tales profesionales, promoviendo la división, principalmente, entre los que producen y los que imparten conocimientos. En este sentido, en lugar de contribuir a la elevación del nivel de los cursos, el Bachillerato provocaría el empobrecimiento del área, producto de la formación especializada (Borges, 2001).

En medio de esa discusión, otro factor tornó el debate más complejo: en virtud del alcance del campo de actuación del área, en muchos cursos (si no en la mayoría), se adoptó la perspectiva de "licenciatura ampliada", es decir, una formación para profesionales preparados para actuar tanto en el espacio escolar como no escolar. Este hecho puede ser considerado un factor determinante en el proceso histórico sobre la formación de profesores de Educación Física (Benites, Souza Neto y Hunger, 2008). 
Lo que se percibe es que la formación de professores está en la base de los cambios ocurridos en la Educación Física. De la misma forma como ocurría en diferentes cursos de Licenciatura, se hacía evidente la falta de un perfil específico en la propuesta del proceso de formación de sus profesionales. Segun Benites, Souza Neto y Hunger, “(...) la 'imagen’ que quedó consagrada por la crítica sobre el curso de Didáctica dentro del contexto de la Educación Física, era la de que se colocaba un "barniz" pedagógico sobre la formación del bachiller" (2008: 8). En ese enredo, y según los autores:

(...) las directrices de formación de profesores ganaron nuevos soportes el 2001 y 2002, con cambios significativos en términos de caracterizaciones y definiciones de criterios para la formación de profesores y muchas discusiones sobre sus lineamientos, lo que propone una "nueva cara" a los cursos de formación, pensando no más en la calificación que estaba centrada en un conjunto de conocimientos, habilidades y capacidades, sino ampliando ese contexto más allá de la formación humana y contemplando los problemas emergentes como la tecnología, carga horaria, infra-estrutura, cantidad de práctica, entre otros. (Benites, Souza Neto y Hunger, 2008: 9).

Como resultado, dos propuestas delimitaron el área de estudio y el campo de actuación de la Educación Física, proponiendo redimensionar el currículo en el proceso de formación profesional: la primera enfatizaba el profesional de Educación Física capacitado para analizar la realidad social e intervir académica y profesionalmente en las diferentes manifestaciones del movimiento; la segunda enfatizaba el profesor de Educación Física visto como un profesional que también debería estar capacitado, prioritariamente, en el ámbito de la docencia (Benites, Souza Neto y Hunger, 2008).

Este cambio de carácter y de orientación de los currículos en el proceso de formación no es resultado de una transformación repentina del perfil de los profesionales del área, sino refleja los sentidos y significados que la Educación Física asume socialmente (Borges, 2001). Se puede destacar que primaron los cursos de formación de "profesores-instructores" en los años 30, evidenciando la fuerte influencia militar y deportivista asociada a los primeros pasos de la Educación Física en Brasil; o el desarrollo del paradigma de la salud y de la aptitud física, evidenciado en el crecimiento y ampliación de espacios para prácticas corporales y deportivas en las últimas décadas del siglo pasado; o igualmente el proceso de desvalorización de los profesores del área, si observamos la expansión del mercado de trabajo, especialmente en clubes, gimnasios y clínicas. Dicho sea de paso, esa expansión en el mercado de trabajo, ciertamente, es uno de los principales responsables de la crisis en los cursos de formación en relación al perfil del profesional de Educación Física (Borges, 2001).

En ese sentido, aunque haya cierta sistematización de los saberes y de las competencias, lo que aparece con más relevancia es la comprensión de que los saberes son plurales, y que la identidad profesional sólo será constituída por medio de una articulación de los diferentes contenidos. En otras palabras, no se trata de formar un pedagogo, aplicando una "capa de barniz" del área de conocimiento específico, ni de formar un profesor del área de conocimiento específico con algunas “pinceladas" pedagógicas (Benites, Souza Neto y Hunger, 2008).

La cuestión central es que, si la discusión sobre la formación de profesores es siempre compleja, independientemente del área de conocimiento, esa discusión se torna aún más complicada cuando pensamos en un área con un campo de actuación tam amplia como la Educación Física. En este sentido, son diversas las críticas en relación a posibles acercamientos a la formación de profesores en esta área. 
Una crítica antigua, aunque aún pertinente, está directamente relacionada a la comprensión de la práctica en Educación Física, asociada a la ejecución y a la demostración de habilidades técnicas y capacidades físicas que remiten a la história dsiciplinar, remanentes de la militarización y deportivización, al contrario de una comprensión más apropiada de la práctica como un proceso de investigación y una actividad creadora-trransformativa en cuanto elaboración y acción sobre la realidad (Ramos, 2002). Si considerarmos esa segunda comprensión de práctica, es evidente su importancia en la formación de profesores.

La perspectiva de la enseñanza reflexiva indica una prática como un espacio de producción, de transformación y de movilización de saberes que le son propios, o sea, debe ser encarada como una importante y riquísima fuente de conocimiento y, por esto, no es posible separar más al futuro profesional (graduado) de su contacto directo, intenso y coordinado con lo cotidiano de la realidad profesional (Ramos, 2002: 42).

Otras críticas están relacionadas a diferentes aproximaciones adoptadas en la formación de profesores, entre ellas: una formación "mixta", o "2 en 1", direccionada, por ejemplo, en la mencionada Licenciatura ampliada, es decir, un abordage no articulado. Se considera que en tres años se hace un curso y con un año más se complementa otro. En síntesis, la crítica no está relacionada diretamente a la certificación del profesional en la aquisición de habilidades relacionadas al "saber hacer", sino a la falta de equilíbrio entre a competencia técnica, el sentido ético-moral, el compromiso con la comunidad y el conocimiento disciplinar (Benites, Souza Neto y Hunger, 2008).

Por otra parte, la crítica está en un modelo epistemológico que atribuye a la formación universitaria, tanto del licenciado como del bachiller en Educación Física, la adquisición de los conocimientos científicos y técnicos prévios para la práctica, en un primer momento, y la aplicación, en un segundo momento (Borges, 2005).

En en marco de ese modelo, la enseñanza en sala de clases es reducida a la aplicación de los conocimientos producidos por los especialistas (científicos en educación física), en tanto la intervención en educación física fuera de la escuela (por ejemplo, en clínicas deportivas) requiere la presencia de un profesional que tenga una formación exclusivamente científica y técnica (Borges, 2005: 183).

Ante este modelo, los profesores de Educación Física acaban por no reconocerse, pues subvaloran su própria formación y, lo más grave, la restringen a un status quo atletaprofesor/profesor-atleta.

\section{EL CURSO DE EDUCACIÓN FÍSICA DE LA UFSCAR: UN POCO DE HISTORIA}

En 1994, se inicia el Curso de Educación Física y Motricidad Humana ${ }^{3}$ de la Universidad Federal de São Carlos (CEFMH/UFSCar), motivado, de una parte, por la situación económica deficitaria que se encontraba la Fundación Educacional São Carlos - FESC- (institución de caráter mixto, de derecho privado con subsídio público de la

Entre 1994 y 2001, el curso tuvó la denominación de "Educación Física y Motricidad Humana". Tal nomenclatura fue alterada por "Educación Física" despues de indicaciones de la Comisión de Especialistas de la Secretaría de Enseñanza Superior del Ministerio de Educación al visitar el referido curso para su reconocimiento, conforme se publicó en DOU de 14/04/2001, sección 1, p. 09 (Ramos et al., 2008). 
Prefeitura Municipal de São Carlos), que desde 1979 manifestaba interés en la abertura de los cursos de Biblioteconomía y de Educación Física en la UFSCar, la cual firmó el compromiso de providencias el 27 de septiembre de 1989, a través de la declaración conjunta del entonces prefecto municipal Neurivaldo José de Guzzi y del entonces Rector de la UFSCar, Prof. Dr. Sebastião Elias Kuri (Universidade Federal de São Carlos, 1989). Por otro lado, concomitante a tal situación existía una “(...) política del gobierno federal de incentivo a la implantación de cursos nocturnos en las universidades públicas brasileiras" (Freitas, Oliveira y Carvalho, 1996: 2).

Así, en 1994, los alumnos aceptados en el proceso selectivo se matricularon en la primera promoción del CEFMH/UFSCar:

Permitiendo atender a los estudiantes ya vinculados al curso fueron aprobadas las disciplinas del primer semestre del marco curricular hasta entonces formulado. (...) En el segundo semestre, el lineamiento en relación al curso se dio de la misma forma. Entre tanto, fue constituída una comisión de profesores de varios departamentos con el objetivo de sistematizar una nueva propuesta para el curso de graduación en EFMH, coherente con la perspectiva "innovadora" de la UFSCar (Freitas, Oliveira y Carvalho, 1996: 2-3).

Tal comisión quedó responsable de una tarea, un tanto complicada, pues, “(...) el tiempo estipulado para la conclusión de los trabajos no podía exceder a 10 meses, período máximo hasta el inicio de las clases de la primera cohorte par matricularse en el curso del año siguinte" (Freitas, Oliveira y Carvalho, 1996: 2-3).

Al igual que ese carácter de urgencia, la propuesta de implantación del CEFMH/ UFSCar fue concluída y, en relación a la formación profesional, quedó establecido que:

La Comisión juzga importante que la dimensión pedagógica oriente la formación del profesional de EF \& MH, toda vez que tal dimensión no se restringe al carácter escolar de la atuación profesional, se tiene en cuenta el aspecto pedagógico que caracteriza también la actuación del Bachiller. Coherente con ese entendimento la UFSCar propone ambas opciones -Bachillerato y Licenciatura- con una FORMACIÓN COMÚM, pues son las especificidades contempladas a través de las disciplinas ofrecidas en los últimos semestres, enfatizando, en ese momento, las características profesionales de cada opción (Universidade Federal de São Carlos, 1995: 6).

La estructura curricular, de acuerdo a las orientaciones de la resolución del Consejo Federal de Educação (CFE) n $n^{\circ} 03 / 87$, quedó entonces compuesta por 3 años básicos y comunes a todos los graduandos, mientras en el $7^{\circ}$ semestre del curso, el alumno debería optar por uno de los dos énfasis oferecidos: Licenciatura o Bachillerato, siendo posible, después de término del $1^{\text {er }}$ énfasis (4 años), habilitarse en el otro cursando 2 semestres más, es decir un $5^{\circ}$ año.

De este modo, las disciplinas específicas ofrecidas para los respectivos énfasis, fueron:

- Licenciatura: Práctica de Enseñanza y Estadías Supervisadas en Educación Física I (Teórica y Práctica); Recursos y Medios para la Enseñanza de Educación Física (Teórica y Práctica); Didáctica en Educación Física (Teórica y Práctica); Educación y Sociedad (Teórica); Monografía; Práctica de Enseñanza y Estadías Supervisadas en Educación Física II (Teórica y Práctica); Estructura y Funcionamiento de la Enseñanza de $1^{\circ}$ y $2^{\circ}$ Grado (Teórica).

- Bachillerato: Estadías III (Práctica); Ciencia y Tecnología en EFMH (Teórica); Fisiología del Ejercicio (Teórica y Práctica); Monografía; Estadía IV (Práctica); Teorías del Entrenamiento en EF (Teórica y Práctica). 
Analizando las características de las disciplinas de Licenciatura y del Bachillerato, es visible que la orientación de la Licenciatura estaba en la preparación de profesores para las escuelas de Educación Básica, mientras el Bachillerato en el conocimiento tecnológico y biológico, en la elaboración de programas de ejercicios y de entrenamiento deportivo, en el ambiente no escolar.

El 22 de enero del 2003, frente a la nueva legislación (Conselho Nacional de Educação. Brasil, 2002a, 2002b) que pasó a exigir integralidad de todos los cursos de Licenciatura (es decir, opción desde el inicio hasta la conclusión de la formación específica), así mismo, y dado el número reducido ${ }^{4}$ de profesores para impartir los dos cursos distintos y paralelos (Licenciatura y Bachillerato), se decidió en la $59^{\mathrm{a}}$ reunión del Consejo del Departamento de Educación Física y Motricidad Humana, la suspensión temporal del Bachillerato y la restructuración curricular del curso de Licenciatura, que proponía, a partir del 2004, la ampliación del período de clases para el vespertino, además de las clases ya existentes en el período nocturno, en los dos últimos años del curso (Universidad Federal de São Carlos, 2003). Tales decisiones fueron aprobadas por unanimidad del citado Consejo Departamental, posteriormente refrendadas por el Consejo de Enseñanza, Investigación y Extensión de la UFSCar del 28 de mayo del 2004 y dispuestas en la Decreto GR nº 765/04 (Universidad Federal de São Carlos, 2004).

Solamente en el 2011, ocurre la aprobación del retorno del curso de Bachillerato en Educacion Física en la UFSCar, con previsión de oferta de 10 cupos para ingreso en el año siguiente, reduciéndose las vacantes de 40 cupos del curso de licenciatura a 30 y contando los dos primeros años de cada curso con disciplinas comunes y los dos últimos con disciplinas específicas, seleccionadas por el candidato a estudiante de la Universidad ya en el proceso de examen de admisión (Universidad Federal de São Carlos, 2011).

Se tiene, entonces, en la história del curso de Educación Física de la UFSCar, tres situaciones:

- entre 1994 y 2003: momento en el cual había oferta conjunta de los énfasis, Licenciatura y Bachillerato, con 40 vacantes disponibles para el estudiante en el ingreso al tronco común del curso y libre opción por un u otro énfasis al final del $6^{\circ}$ semestre del curso.

- entre 2004 y 2011: oferta exclusiva de Licenciatura en Educación Física (40 cupos).

- 2012 a la actualidad: posibilidad de elección del curso de Licenciatura (30 cupos) o de Bachillerato (10 cupos), en que el estudiante indica la opción por el curso de formación en Educación Física, ya en el acto de inscripción al Sistema de Selección Unificada -SiSU(Ministério da Educação. Brasil, 2010).

En este estudio, nos concentramos en promociones de estudiantes recien ingresados de dos de esos momentos del curso, a saber, de tres cohortes del segundo momento, específicamente cohortes del 2006, 2007 y 2008, y de la primera cohorte del tercer momento: 2012.

El Departamento de Educación Física y Motricidad Humana (DEFMH) contaba, en ese tiempo, con 12 docentes efectivos, no obstante cuatro de ellos se encontraban con permiso parcial por estudios de doctorado y uno en permiso completo por estudios de pos-doctorado. Vale destacar que el Departamento de Educación Física en Universidades Públicas Brasileiras acostumbra a tener más de 20 docentes efectivos. Destacamos además que el DEFMH es el departamento con menor número de docentes, inclusive en la UFSCar, pues ésta considera 15 el número mínimo de docentes para la constitución de un Departamento, por lo cual el DEFMH se considera un Departamento en carácter de excepción. 


\section{TRAYECTORIA METODOLÓGICA}

En busca de develar la comprensión de los estudiantes de primer semestre sobre las características de la Licenciatura en Educación Física, analizamos relatos de las cohortes ingresadas los años 2006, 2007, 2008 y 2012, registrados el primer día de clases de la disciplina Dimensiones Socio-antropológicas de la Educación Física, administrada por uno de los autores de este estudio, del curso de Licenciatura en Educación Física de la UFSCar.

Como instrumento de registro fue utilizado un cuestionario de una pregunta abierta, (Negrine, 1999): “¿Por qué usted escojió el Curso de Licenciatura en Educación Física?”, que los estudiantes respondieron por escrito en hojas anexas. Las respuestas resultantes fueron sometidas al análisis cualitativo, del tipo análisis de contenido temático, en el cual, según Gomes (2011), se realiza un proceso de categorización en función de establecer clasificaciones agrupando elementos, ideas o expresiones, cuyo eje central es el tema. El mismo autor plantea que la categorización puede ser realizada tanto previamente, como surgir a partir del análisis del material de investigación, siendo este último el procedimento adoptado en el presente estudio.

Para la construcción de las categorías utilizamos una cantidad de relatos de las cohortes arriba mencionadas y que se distribuyen de la siguiente forma: 2006 (37 estudiantes), 2007 (36 estudiantes), 2008 (39 estudiantes), quienes sólo tenían como opción la Licenciatura en tanto posibilidad de formación en Educación Física en la UFSCar, pues el Bachillerato estuvó suspendido en esta universidad hasta 2011.

\section{PRESENTACIÓN DE LOS RESULTADOS}

\subsection{CATEGORÍA A: NO IDENTIFICACIÓN CON LA ENSEÑANZA ESCOLAR}

Entre las exposiciones de los estudiantes destacamos las taxativas, asociadas a la no intensión de trabajar en el contexto escolar: "No pretendo dar clases" (estudiante 8, cohorte 2006); “[...] no pretendo trabajar en la Educación Física escolar" (estudiante 18, cohorte 2006); "[...] no pretendo seguir la carrera de profesor, dando clases" (estudiante 25, cohorte 2006).

De igual forma, destacan aquellas exposiciones en que los discentes relevan que el sentido de su elección se basa en el gusto por el deporte, entrenamiento y competición, resaltando la vivencia previa de tales aspectos relacionada a prácticas deportivas fuera del ambiente escolar y/o intencionalidad de actuar en deportes y gimnástica en clubes y gimnasios: “(...) siempre me gustó praticar deportes” (estudiante 37, cohorte 2006); “(...) siempre practiqué algún deporte" (estudiante 3, cohorte 2007); “(...) siempre me interesé por los deportes” (estudiante 4, cohorte 2007); “(...) amo practicar deportes, ya hice un poco de casi todo" (estudiante 28, cohorte 2008). En tanto, el deporte al cual se están refiriendo es, principalmente, el de rendimiento: “(...) viajaba a las competiciones” (estudiante 2, cohorte 2008); “(...) jugaba fútbol en clubes que contaban con categorías de base, y mis entrenadores apostaban por mi fútbol” (estudiante 11, cohorte 2008); “(...) disfrutar de deportes de alto rendimento, con bicicletas" (estudiante 37, cohorte 2008); “(...) disputaba campeonatos representando a la escuela y a la ciudad" (estudiante 5, cohorte 2007); “(...) siempre practiqué varios deportes y, competitivamente, basquetbol" (estudiante 22, cohorte 2007); "Me gustan los deportes y competiciones, inclusive el fútbol" (estudiante 30, cohorte 2007). 
Hacer el curso de Licenciatura en Educación Física remite, para los entrevistados, a la continuidad da práctica deportiva: “(...) decidí hacer Educación Física porque es un curso vinculado al deporte en general” (estudiante 18, cohorte 2007); “(...) ingresar a este curso me hace querer volver a jugar handbol y nadar, que son deportes que deje de practicar hace unos diez años" (estudiante 20, cohorte 2008); "Ví en el curso de Educación Física una manera de no abandonar el basquetbol” (estudiante 39, cohorte 2008); “(...) quisiera continuar entrenando, compitiendo" (estudiante 9, cohorte 2006). Consecuentemente, la continuidad está asociada al trabajo en la modalidad con que tuvieron contacto: "También quisiera trabajar como técnico o preparador físico en el área del tenis" (estudiante 8, cohorte 2008); “(...) no olvido el fútbol, por eso, me gustaría desempeñarme en la preparación física de jovenes (clubes de fútbol que cuenten con categorías de base)" (estudiante 11, cohorte 2008).

Otra indicación muy recurrente en esta categoría fue el trabajo en gimnasios y clubes, específicamente en el entrenamiento y la rehabilitación: "[...] pretendo trabajar como personal trainer" (estudiante 18, cohorte 2006); "Pienso en trabajar en gimnasios o clubes" (estudiante 19, cohorte 2006); “(...) tengo como objetivo principal la preparación física" (estudiante 7, cohorte 2007); “(...) entrenamientos y nutrición deportiva son algunas áreas que me interesan de la Educación Física y en las cuales me gustaría trabajar" (estudiante 13, cohorte 2007); "Me gusta también el área da danza y trabajar con rehabilitación" (estudiante 21 , cohorte 2008); “(...) tengo ganas de trabajar en rehabilitación en todos los aspectos" (estudiante 22, cohorte 2008); “(...) trabajar con rehabilitación de personas obesas” (estudiante 24, cohorte 2008); “(...) relacionar el ballet clásico y así ayudar en los problemas ocasionados por la práctica a largo plazo del mismo” (estudiante 11, cohorte 2006).

\subsection{CATEGORÍA B: MOTIVOS PARA LA (FALTA DE) ELECCIÓN PROFESIONAL}

Esta categoría presenta consideraciones en relación a tener o haber tenido otra pretensión de estudio que no sea la Licenciatura en Educación Física.

Las primeras indicaciones son relacionadas al curso de Licenciatura en Educación Física como segunda opción: "En el examen de ingreso (Vestibular) esta no fue mi primera opción" (estudiante 25, cohorte 2006); "Yo elegi licenciatura como segunda opción" (estudiante 8, cohorte 2006). Entre los motivos que les llevaron a tomar la segunda opción están: no conseguir mejor resultado en el examen de seleccion - "Siempre quise Ingeniería, pero no conseguí pasar" (estudiante 35, cohorte 2006); mayor número de candidatos por vacantes - "Entonces intenté algún curso mas fácil de pasar" (estudiante 35, cohorte 2006); la universidad no tiene el curso pretendido “(...) por no encontrar mi primera opción que era Farmacia" (estudiante 23, cohorte 2008); mayor número de vacantes "Yo pretendía ingresar al curso de bachillerato, pero debido a dificuldades como el número de vacantes reducido ${ }^{5}$ (...) opté por el curso de licenciatura" (estudiante 2, cohorte 2012); mayores posibilidades de atuación profesional “(...) pensé en postular a danza también, pero la facultad de danza no me abre tantos caminos diferentes como la Educación Física” (estudiante 25, cohorte 2008); menor exigencia de estudios "Educación Física no es un curso que exiga tanto como

Como ya fue indicado anteriormente, existían sólo 10 vacantes en el curso de Bachillerato contra 30 del curso de Licenciatura, en el caso de la UFSCar. 
Ingeniería, por ejemplo" (estudiante 34, cohorte 2008); lógica SiSU6 “Infelizmente, mi rendimiento en el ENEM fue bajo de lo esperado y mi pontuación fue suficiente solo para la aprobación en Educación Física (...) Mi aprobación fue para la licenciatura, pero yo quería el bachillerato" (estudiante 27, cohorte 2012); "Aunque esté matriculado en licenciatura, por el área que me gusta debería estar haciendo bachillerato, pero por la dificuldad en la puntuación del examen de selección acabé cursando la licenciatura" (estudiante 29, cohorte 2012); "Coloqué en $1^{\text {a }}$ opción, pues la Fisioterapia estaba muy lejos, y llegue a la licenciatura. En verdad, quisiera haber postulado al bachillerato, pues encuentro que tiene más que ver conmigo" (estudiante 32, cohorte 2012):

(...) sólo me restaba el SiSU, pero no iba a colocar Medicina pues la competencia continuaría siendo grande y mis chances diminuirían aún más. Decidí colocar Fisioterapia como primera opción y Ciencias Biológicas como segunda. Pero despues mi ubicación fue cambiando en esos cursos y en el último día cambié a Educación Física como primera opción (...) fui llamada en la $5^{a}$ lista y no dudé en inscribirme (estudiante 35, cohorte 2012).

Como primera opción estaban: Administración; Bachillerato en Educación Física; Biología; Danza; Enfermería; Ingeniería; Farmacia; Fisioterapia; Imagen y Sonido; Letras; Nutrición, Medicina, Ciencias Biológicas, Derecho y Piloto Militar.

Otra realidad encontrada fue la de estudiantes que ya terminaron o iniciaron otros cursos. Los cursos fueron: Ciencias Biológicas; Ciencias Exactas; Ingeniería Civil; Letras; Medicina Veterinária y Química.

Los motivos para iniciar una segunda graduación fueron: no identificarse como un profesional del área “(...) no me ví como un profesional de esa área” (estudiante 27, cohorte 2008); cursar una graduación en el área de biología y de educación "Escogí hacer Educación Física, pues encontré interesante hacer una segunda graduación en el área de biología y de la educación" (estudiante 20, cohorte 2007); sentir falta de actividades físicas "me gusta la profesión del área biológica, pero siento falta de actividades físicas" (estudiante 27, cohorte 2007).

Otro foco indicado está relacionado con la carrera académica, especialización, mestría, doctorado y docencia, en la enseñanza superior: “(...) pretendo hacer Maestría y Doctorado” (estudiante 29, cohorte 2006); “(...) si lo logro, después de terminar el curso, me especializaré en las áreas de entrenamientos específicos y preparación física” (estudiante 34, cohorte 2007); “(...) profesor (...) tal vez en una universidad” (estudiante 8, cohorte 2008); "Tengo en mente trabajar en el campo de investigación de la Educación Física, quizas cursar un magíster o hasta un doctorado y volverme un profesor de Universidad" (estudiante 14, cohorte 2008); “(...) haciendo un curso de especialización” (estudiante 33, cohorte 2008).

La intención de hacer el curso para conocerlo, saber sus posibilidades de actuación, también es expuesta por los estudiantes: “(...) conocer el curso de Educación Física para

El proceso selectivo del SiSU posee una única etapa de inscripción. Al efectuar la inscripción, el candidato escoje, por orden de preferencia, hasta dos opciones entre los cupos ofrecidos por las instituciones participantes del SiSU. Es permitido al candidato, durante el período de inscripción, modificar sus opciones cuantas veces quiera, siendo considerada valida la última inscripción confirmada. Al final de la etapa, el sistema selecciona automáticamente los candidatos mejor clasificados en cada curso, de acuerdo con sus notas en ENEM. Son considerados seleccionados solamente los candidatos clasificados dentro del número de cupos ofrecidos por el SiSU en cada curso. Los candidatos no seleccionados en ninguna de sus opciones en las llamadas regulares y los candidatos seleccionados en su segunda opción, pueden participar de la Lista de Espera de SiSU (Ministério da Educação. Brasil, 2013). 
saber lo me puede ofrecer” (estudiante 27, cohorte 2006); “(...) bueno, iniciando el curso pretendo ver si la Educación va a ser en lo que yo quiero trabajar en mi vida" (estudiante 25, cohorte 2007); "Aún no tengo una noción de en qué área específicamente quiero trabajar" (estudiante 9, cohorte 2008); “(...) aún no tengo mucha idea sobre mi futuro en la carrera” (estudiante 15, cohorte 2008).

La pretensión de ampliar posibilidades de actuación debido la dificultad encontrada en la inserción en el mercado de trabajo fue encontrada en las respuestas de los estudiantes: “(...) escojer la licenciatura se dió por saber de la dificultad que el profesional de la Educación Física encuentra muchas veces en el mercado" (estudiante 20, cohorte 2006).

\section{CONCLUSIONES}

Después de la exposición de algunos aspectos de la formación profesional en Educación Física, incluyendo un ejemplo situado en la experiencia vivida en un curso de Licenciatura en el área, algunos puntos relacionados con (posibles) motivos de la caracterización erronea de ésta precisan ser destacados.

Lo primero es la lógica de "Licenciatura ampliada" que, en teoría, prepararía profesionales para actuar en el espacio escolar y no escolar al mismo tiempo, lo que puede ser interpretado como falta de especificidad de cada actuación y, al mismo, tiempo un déficit en la formación situada en cada campo de desempeño profesional. En este contexto, la misma práctica profesional fuera de los límites escolares sería fácilmente reproducida en el ambiente escolar, lo que justificaría el énfasis en la preparación física de individuos, a través de la sistematización de entrenamientos y de prácticas deportivizadas. Este aspecto puede ser una de las causas de la visión de un profesor más cercano a una tipificación de entrenador, lo que reduce su actuación y dimensión política en el sentido crítico, más bien se orienta hacia procesos conservadores y cercanos a enteder la actividad de ejercitación como ajena a la consciencia política y cercana a los criterios de cosumo propios del deporte espectáculo. Tampoco se debe olvidadar la aproximación y evidencia histórica entre militarismo y Educación Física, con su sesgo de salud y aptitud física que lleva hoy en día a compreder que un buen profesor es quien mantiene el control y disciplina propia de la estructura militar, donde la sanción incluso se presenta con los propios contenidos de la asignatura.

En este enfoque, el aspecto biológico y fisiológico de los individuos se tornan coherentes a las acciones de demostración y ejecución técnica, preferentemente sin mayores cuidados interpersonales, para no interferir en los ciclos de entrenamientos. Dentro de este paradigma, el desarrollo de una actitud crítica, de la reflexión y de la autonomía es coherentemente abandonado, pasando el profesor a ser visualizado como um instructor o como atletaprofesor/profesor-atleta y su interés fundamental está en la reproducción acrítica de modelos estandardizados y relaciones de poder ya establecidas de dominación y control.

Mas allá de los equívocos epistemológicos, lo que se presentó como importante, a partir de las comprensiones de discentes de un programa de formación de profesores, fue una cuasi rechazo a la Educación Física en el contexto escolar, que en teoría, según la Resolución CNE/CP 1/2002, que instituye las Directrices Curriculares Nacionales para la Formación de Profesores de la Educación Básica, en nivel superior, curso de Licenciatura, de graduación plena (Conselho Nacional de Educação. Brasil, 2002a), incluyendo, consecuentemente, la Licenciatura en Educación Física, el objetivo general de los cursos de Licenciatura es la 
formación de un profesional competente, socialmente crítico y responsable por los destinos de una sociedad que se desea justa, democrática y autosustentable.

El objetivo fundamental de los cursos de Licenciatura, entonces, es formar licenciados como sujetos de transformación de la realidad brasileña, comprometidos con la búsqueda de respuestas a los desafíos y problemas existentes en nuestras escuelas, especialmente en las pertencientes a la red pública. Desde allí surge un conjunto de objetivos que deberían orientar la formación del licenciado: comprender el contexto de la realidad social de la escuela brasileña (sus valores, representaciones, historia y prácticas institucionales) de modo que pueda asumir una postura crítica y responsable de transformación de esa realidad; orientar sus opciones y decisiones profesionales por princípios éticos, por la superación de prejuicios, por la aceptación de la diversidad de las/os alumnas/os partiendo del entendimento de que toda/o alumna/o es capaz de aprender, independientemente de la condición social a la que pertence; comprender los procesos de enseñanza y aprendizaje (re) construyendo los saberes disciplinares y las actividades de enseñanza; crear, implementar, evaluar y perfeccionar proyectos de enseñanza y aprendizaje y articularlos con otras áreas del conocimiento, estimulando en la escuela acciones colectivas y multidisciplinares, de modo que permitan caracterizar, así, un nuevo éthos profesional; investigar el contexto educativo en su complejidad y analizar su práctica profesional (Conselho Nacional de Educação. Brasil, 2002a).

Reforzando la comprensión de fuga del contexto de la Licenciatura, surge la indicación, sistematizada desde las respuestas de los discentes investigados, de que el curso no estaba en los planes de formación y acabó "materializándose" como una suerte de "castigo" o "mal menor" por no haber conseguido ingresar en la primera opción deseada.

El curso de Licenciatura se configura, en este contexto, más como falta de opción que, realmente, como una elección profesional. La menor concurrencia, la supuesta menor exigencia de estudios, la supuesta posibilidad de continuidad de prácticas deportivas y ampliación de empleabilidad, fueron motivos indicados por los estudiantes recién ingresados para estar en la enseñanza superior de Licenciatura en Educación Física. Ponemos en tensión que la especificidad de la Licenciatura y la búsqueda de la calidad de la enseñanza en todo el sistema educativo, en especial en la Educación Básica, corre riesgos en la situación de que tal conducta de los estudiantes novatos permaneciera durante todo su formación profesional e ingreso al trabajo del ambiente escolar. Este es un aspecto muy interesante que lleva a cuestionarse sobre los procesos de formación docente una vez que los futuros profesionales están dentro de los programas y registrar los procedimientos que los responsables de dicha formación elaboran para dar cumplimento con una formación crítica y transformadora. Por lo cual, conforme a lo ya enfatizado con Tardif y Raymond (2000), los saberes relacionados a lo que es y significa ser docente también serán movilizados con la propia formación académica y, siendo así, podrán ser positivamente alterados en el proceso de formación y en el inicio de la carrera.

El problema mayor se da cuando los docentes formadores aceptan el papel de "meros" reproductores de instrucciones, lo que ciertamente colabora en la perpetuación del status quo equivocado de la Licenciatura en Educación Física, y lo que es aún más grave, con el status quo social y cultural de ésta, rechazando de plano los procesos de transformación y democracia que una socidedad actual requiere.

Es importante resaltar que en el imaginario de los estudiantes novatos se revela una comprensión disciplinar que precisa ser analizada y estudiada por los centros de formación 
Estudios Pedagógicos, vol. XL, Número Especial 1: 87-103, 2014

FORMACIÓN DE PROFESORES EN EDUCACIÓN FÍSICA EN BRASIL: COMPRENSIÓN DE ESTUDIANTES

NOVELES DE LA UNIVERSIDAD FEDERAL DE SAN CARLOS

de docentes, pues claramente hay un mensaje muy alejado de una disciplina comprometida con procesos de construcción y desarrollo social que impliquen tanto la denuncia como el esfuerzo transformador de las condiciones sociales y culturales. Al lo menos al ingreso, esta disciplina se visualiza como un mal menor dentro de la construcción de un proyecto personal, pero muy lejos de un proyecto social o cultural. A nuestro modo de ver, eso no es una casualidad, sino que tambien responde a los mensajes y posicionamento de la disciplina dentro de la sociedad.

Por último, entendemos que estudios con estudiantes egresados, al inicio de la carrera profesional, deben ser realizados para profundizar en la comprensión tanto de la formación como de la actuación de profesores de Educación Física en el contexto escolar y sus posibilidades de trasnformación en situaciones específicas, como también en marcos legales y culturales que favorezcan o limiten su desempeño laboral.

\section{REFERENCIAS BIBLIOGRÁFICAS}

Andrade Filho, N.F. (2001). Formação profissional em Educação Física brasileira: uma súmula da discussão dos anos de 1996 a 2000. Revista Brasileira de Ciências do Esporte, vol.22, n.3, 23-37.

Barros, J.M.C. (1995). Educação Física na UNESP de Rio Claro: bacharelado e licenciatura. Revista Motriz, vol.1, n.1, 71-78.

Benites, L.C., Souza Neto, S. y Hunger, D. (2008). O processo de constituição histórica das diretrizes curriculares na formação de professores de Educação Física. Educação e Pesquisa, vol.34, n.2, 343-360.

Betti, I. y Betti, M. (1996). Novas perspectivas na formação profissional em Educação Física. Revista Motriz, vol.2, n.1, 10-15.

Betti, M. (2009). Educação Física e sociedade: a educação física na escola brasileira (2a ed.). São Paulo: Editora Hucitec.

Borges, C.M.F. (2005). A formação dos docentes de Educação Física e seus saberes profissionais. En Borges, C.M.F. y Desbiens, J.F. (Orgs.), Saber, formar e intervir para uma Educação Física em mudança (pp. 157-190). Campinas: Autores Associados.

Borges, C.M.F. (2001). O professor de Educação Física e a construção do saber (2a ed.). Campinas: Papirus.

Conselho Nacional de Educação. Brasil. (2002a). Resolução CNE/CP 1, de 18 de fevereiro de 2002. Brasília: MEC/CNE.

Conselho Nacional de Educação. Brasil. (2002b). Resolução CNE/CP 2, de 19 de fevereiro de 2002. Brasília: MEC/CNE.

Ministério da Educação. Brasil. (2013). Sistema de seleção unificado: como funciona? Última visita 2 julio de 2013. Recuperado desde http://sisu.mec.gov.br/como-funciona

Ministério da Educação. Brasil. (2010). Ministério da Educação. Secretaria de Educação Superior. Portaria Normativa ${ }^{\circ}$ 2, de 26 de janeiro de 2010. Brasília: MEC/Sesu.

Comissão de Especialistas de Ensino em Educação Física. (1999). Proposta de diretrizes curriculares: curso de graduação em Educação Física. Brasília: COESP/EF.

Freitas, F.F., Oliveira, P.E.O. y Carvalho, Y.M. (1996). Escrevendo a história do Curso de Educação Física e Motricidade Humana na perspectiva do aluno. São Carlos: UFSCar.

Gomes, R. (2011). A análise de dados em pesquisa qualitativa. En M.C. Mynaio (Org.), Pesquisa social: teoria, método e criatividade (30a ed.) (pp. 79-108). Petrópolis: Vozes.

Gonçalves Junior, L., Ramos, G.N.S. y Machado, D.F.V. (2001). Formação profissional em Educação Física no Brasil: o velho problema do currículo e o caso da UFSCar. En Anais do 
Congresso Estadual Paulista Sobre Formação de Educadores: Formação de Educadores - Desafios e Perpectivas para o Século XXI, 6. Universidade Estadual Paulista. Águas de Lindóia, São Paulo.

Negrine, A. (1999). Instrumentos de coleta de informações na pesquisa qualitativa. En V. Molina Neto y , A.N.S Triviños (Orgs.), A pesquisa qualitativa na Educação Física: alternativas metodológicas (pp. 61-93). Porto Alegre: UFRGS/Sulina.

Oliveira, A.A.B. y Costa, L.P. (1999). Educação Física/esporte e formação profissional/campo de trabalho. En S.V. Goellner (Org.), Educação Física/Ciências do Esporte: intervenção e conhecimento (pp. 83-99). Florianópolis: Colégio Brasileiro de Ciências do Esporte.

Oliveira, J.G.M., Betti, M. y Oliveira, W.M. (1988). Educação Física e o ensino de $1^{o}$ grau. São Paulo: EPU/EDUSP.

Ramos, G.N.S. (1995). Educação Física: licenciatura elou bacharelado? analisando as implicações de uma estrutura curricular (Dissertação Mestrado em Educação, Programa de PósGraduação em Educação). Pontifícia Universidade Católica de São Paulo, São Paulo.

Ramos, G.N.S. (2002). Preparação profissional em Educação Física: a questão dos estágios (Tese Doutorado em Educação Física, Programa de Pós-Graduação em Educação Física). Universidade Estadual de Campinas, Campinas.

Ramos, G.N.S., Gonçalves Junior, L., Paschoalino Junior, L.C., Santos, L.C. y Andrade, M.C.R. (2008). Egressos do curso de educação física da Universidade Federal de São Carlos (1997-2003): formação e atuação. Movimento \& Percepção, vol.9, 249-265.

Tardif, M. y Raymond, D. (2000). Saberes, tempo e aprendizagem do trabalho no magistério. Educação \& Sociedade, vol.21, n.73, 209-244.

Tojal, J.B. (1995). Currículo de graduação em Educação Física: a busca de um modelo. Campinas: Ed Unicamp.

Universidade Federal de São Carlos. (1989). Declaração do Prefeito Municipal e do Reitor da Universidade Federal de São Carlos em relação aos cursos da Fundação Educacional do Município. São Carlos: Autor.

Universidade Federal de São Carlos. (1995). Comissão de Professores. Proposta de implantação do Curso de Educação Física e Motricidade Humana. São Carlos: Autor.

Universidade Federal de São Carlos. (2003). Ata da 59a Reunião Ordinária de Conselho do $D E F M H$. Conselho/DEFMH, de 22 de janeiro de 2003. São Carlos: Autor.

Universidade Federal de São Carlos. (2004). Portaria GR $n^{\circ}$ 765/04. Dispõe sobre alterações no curso de educação física. São Carlos: Autor.

Universidade Federal de São Carlos. (2011). Ata da $33^{a}$ Reunião Ordinária de Conselho do CCBS. Conselho/CCBS, de 22 de novembro de 2011. São Carlos: Autor. 
